\title{
Advances in the Psychological Assessment of Offender Patients
}

\author{
By D. A. Black, Consultant Clinical Psychologist, Broadmoor Hospital
}

Psychological assessment at Broadmoor in the 20 years lifetime of the Psychology Department has been directed at the description of patterns of response indicative of abnormalities associated with diagnostic and offender categories and suggestive of treatment needs. The results have also frequently been accumulated for research purposes, particularly to identify any common patterns associated with treatment outcome and with discharge prediction and outcome.

Research and clinical usage have resulted in an evaluation of the various assessment methods for offender patient purposes, which may be summarized according to the functions being assessed, as follows:

1. Cognitive Functions (e.g. I.Q. and specific ability tests): No specific patterns relevant to offender patients but results relate to the usual variations of impairment associated with psychiatric nosology and help to identify, for example, educational needs and occupational potential.

2. Perceptuo-motor Functions (e.g. visual tracking, drawing, mazes): Such tasks have traditionally been found to be sensitive to the effects of impulsive, rapid and careless variation in motor control. To the extent that such variation is frequently typical of some offender groups (trends confirmed in our population), maze-type tasks are useful in the description of offender-patients.

3. 'Personality' (a variety of methods intended to record individual differences in behaviour, temperament etc):

a) Questionnaires:-These have proved useful in the offender-patient setting to identify 'personality types' associated with various kinds of offence, e.g. domestic homicide and habitual non-homicidal personal violence (Blackburn, 1971, 1974). Different histories, victim targets and habitual behavioural 'styles' tend to distinguish these two groups, the first showing characteristics of excessive inhibitory controls, denial and repression, whereas the nonhomicidal aggressor tends to show impulsiveness and acknowledge hostility.

b) Projective Methods:-These fail to show responses specific to offender patients. Marked differences in response occur but seem unrelated to overt behaviour.

c) Rating Scales:-These have their use in reflecting changes in behaviour in specific settings, e.g. nurse observation of ward behaviour and occupation officer observations of work behaviour. d) Repertory Grids/Semantic Differentials:-These deserve mention because they combine objective, analysable 'scores' with the projective element of the individual's own experience and value system. Whilst, again, no specific typology is demonstrable in offender patients, considerable work has been done with these methods to map out perceptions and cognitive evaluations that characterize an individual's view of and reaction to his personal world and the problems within it. An example of this approach is the work of Howells and Wright (1978).

4. Physiological and Biochemical Variations (e.g. variations in pulse, breathing, skin conductance, muscle tension, penile tumescence and the constituents of body fluids resulting from psychological events such as perceived stress, sexual interest etc.): Meaningful changes have been identified with such psychological events in offender patients and have helped to identify areas of sensitivity and abnormal arousal patterns. These are helpful in indicating treatment needs and response to treatment (e.g. Crawford, 1979) but the implications for events outside the laboratory remain to be established.

5. Social Behaviour and Interaction (e.g. behaviour analysis, psychological history taking and video or audio recordings of behavioural responses to representative situations): Direct observation and measurement is almost always preferable to measurement of some putative correlate or postulated intermediate process, provided the element of deceit or distortion can be prevented or overcome. These approaches are relevant to the process of explanation and of treatment planning and evaluation but are not conducive to deriving 'norms' and hence the identification of distinct offender-patient groups, (i.e. they are idiographic, not nomothetic).

In summary, work at Broadmoor's Psychology Department has not produced, nor was it expected or intended to produce, 'cook-book' identification of categories of offenderpatients. As with other offender groups and patient situations, a wide variety of offences, precipitating circumstances and psychiatric conditions are encountered, requiring a correspondingly wide range of explanations and care and treatment strategies. A variety of methods, as described above, are in use to help identify those stresses and pressures to which an individual has been exposed or which he perceives as stressful, provoking or attractively arousing. The validity and usefulness of these methods are exemplified in the varied work referred to, and is also testable by looking 
at the post-discharge careers of patients. One such study (Black, 1976) shows the value of the typology described by Blackburn (1971), but more sophisticated studies of treatment and discharge outcome will be required to evaluate fully the variety of other methods which have been developed.

\section{REFERENCES}

BLACK, D. A. (1976) 'A five year follow-up study of male patients discharged from Broadmoor Hospital'. Paper presented to the NATO Advanced Study Institute, Cambridge, July 1976. Available in cyclostyle from author and to appear in Advances in Forensic Psychiatry and Psychology, eds. J. Gunn \& D. P. Farrington. Chichester: John Wiley \& Sons.

\section{Forthcoming Events}

The Third World Congress on Pain, sponsored by the International Association for Study of Pain, will be held from 4 to 11 September, 1981, in Edinburgh. Information: Third World Congress on Pain. University of Edinburgh, 16 George Square, Edinburgh EH8 9LD; or Louisa Jones, Secretary, International Association for the Study of Pain, University of Washington, Seattle, WA 98195, USA.

The next APIT MRCPsych Examination Workshop will be held on 18 and 19 October, 1980. Course fee $£ 24$ (APIT members: £20). Information: Mrs Vanstone-Walker, Course Secretary, Maudsley Hospital, London SE5 8AZ.

A half-day release course in 'Child Psychiatry and Mental Handicap' has been organized as part of the Advanced Level Course for the MRCPsych Examination by the London and St. Bartholomew's Hospitals. It will be held on Wednesday afternoons from 1 October to 17 December, 1980, in the Department of Psychiatry, The London Hospital. Information: Mrs $O$ Weltman, Psychiatric Out-patient Department, The London Hospital, London E1 1BB.

A half-day release MRCPsych Course in Basic Sciences (Part 1), organized by London and St. Bartholomew's Hospitals will be held at the Department of Psychological Medicine, St Bartholomew's Hospital on Wednesday afternoons from 1 October to 17 December, 1980. Information: Mrs C Hawkins, German Hospital, Ritson Road, London E8.
BlackbURN, R. (1971) Personality types among abnormal homicides. British Journal of Criminology, 11, 14-31.

(1974) Personality and the Classification of Psychopathic Disorders. Special Hospitals Research Report No. 10, 119 Camberwell Road, London SE5 OHB.

Crawrord, D. A. (1979) Modification of deviant sexual behaviour: the need for a comprehensive approach. British Journal of Medical Psychology, 52, 151-6.

HoweLLS, K. \& WRIGHT, E. (1978) The sexual attitudes of aggressive sexual offenders. British Journal of Criminology, 18, 2, $170-4$

The views presented in this paper are those of the author and do not necessarily reflect those of the Department of Health and Social Security.
The First International Conference of the Mental Research Institute entitled 'Innovations in Psychotherapy and Family Medicine' will be held from 25 to 27 June, 1982, in Nice. Information: Diana Sullivan, Everstine, MRI, 555 Middlefield Road, Palo Alto, California 94301, USA.

A one-week introductory course in Child and Adolescent Psychiatry has been organized by members of the staff of the Institute of Child Health and the Hospital for Sick Children. The course will be held at the Institute from 24 to 28 November, 1980. Information: The Sub-Dean, Institute of Child Health, 30 Guildford Street, London WCIN 1EH.

A General Course in Group Work will be held on Thursday evenings from October 1980 to June 1981 at the Institute of Group Analysis. Information: The Course Secretary, Institute of Group Analysis, 1 Bickenhall Mansions, Bickenhall Street, London W1H 3LF.

The 8th World Congress of Social Psychiatry will be held in Zagreb from 16 to 22 August 1961. Participation by presentation of papers, symposia and panel discussions is welcome. Information: Dr M. Stojanovic, University Hospital, Vinogradska c. 29, 41000 Zagreb, Yugoslavia. 